\title{
Face Photograph Recognition via Generation from Sketches using Convolutional Neural Networks
}

\author{
Mustafa Karasolak, Roya Chopani \\ Computer Engineering Department \\ Cankaya University \\ Ankara, Turkey
}

\begin{abstract}
Face photo-sketch matching is an important problem for law enforcement agencies in terms of identifying suspects. In this study, a new sketch-photo generation and recognition technique is proposed by using residual convolutional neural network architecture. The suggested RCNN architecture consists of 6 convolutions, 6 ReLU, 4 poolings, 2 deconvolution layers. The proposed architecture is trained with face photos and sketches. Sketches are supplied as an input to the RCNN architecture and, generated face photos are obtained as the output. Then, the generated face photos are compared with the photos of the people in the database. Structural Similarity Index (SSIM) is used to measure the pairwise similarity and the photo with the highest index score is matched. CUHK Face Sketch Database containing 188 images is tested. In the experiments, 148, 20, and 20 images are used for training, validation, and testing, respectively. Data augmentation applied to 148 training images produced 444 images. Experimental results show that the success of the training curve is $90.55 \%$ and the validation success is $91.1 \%$. True face recognition success from generated face images with SSIM is 93.89\% for CUHK Face Sketch database (CUFS) and $84.55 \%$ AR database.
\end{abstract}

\section{Introduction}

Recently, machine learning and image processing methods have been developed. Especially, when the human eye is inadequate and deceptive, machines can make more reliable decisions [1]. Image processing methods that are used primarily for monitoring purposes are used later to facilitate interpretation of scenes. For this purpose, applications such as image segmentation, color change and square fitting are used [2].

Face recognition systems are very important for law enforcement. It is highly efficient in terms of automatic identification of suspects and speeding up of transactions. Generally, the images obtained from color camera sensors are similar to those displayed in the data log. It is relatively easy to solve such problems. In some cases, images of ordinary suspects obtained from RGB camera sensors cannot be obtained. In such cases, sketch drawing is used. Sketch drawings of the same person are quite different when drawn by different people. For this reason, if sketch drawings are converted to RGB images, identification of the person becomes easier.

In this study, a residual CNN structure is proposed to obtain RGB face images using sketch drawing. CNN structures have a very suitable architecture for image processing problems and are used in many studies in the literature [3]. This architecture, which is famous after [4] Krizhevsky and his colleagues won the Imagenet competition using the CNN structure [4], is used almost all image and video problems thanks to the convolution layer's suitability for image processing problems. CNN architecture automatically learns features from the image. Compared to handcrafted features, automatic feature learning is quite successful. Because hand-crafted features require experience, expertise and knowledge. In addition, the specified features may not always represent the data successfully. For this reason, algorithms that can learn automatic features come to the forefront. Many studies on face recognition and sketch identification problems have used hand-crafted features. Tang et al. [5] used geometric features and eigenface vectors for face sketch recognition. Liu et al. [6] applied a mapping study according to geometric similarities between face photograph and sketch. Zhang et al. [7] proposed a coupled information-theoretic encodingbased feature extraction method for automatic facesketch recognition. Galoogahi and Sim [8] propose a new face descriptor based on gradient orientation for automatic face sketch recognition. In applications which are performed with hand-crafted features, images are classified with a hand-selected classifier. But deep learning automatically classifies the features it acquires on its own. Mittal et al. [9] use transfer learning with deep learning representation for sketch recognition. Zhu et al. [10] propose a new deep learning framework that can recover the canonical view. Tang and Wang [11] have compared face sketch images with real images by suggesting an automatic image retrieval system in the CUHK data set. Bansode and Sinha [12] proposed a genetic algorithm-based image generation system from color image to face sketch image. Pramanik and Bhattacharjee [13] used principle component analyze to extract feature from color images. Then, some classifiers, which are KNN and SVM, applied to match face sketch images in CUHK database.

In this study, a highly effective method is proposed for face-sketch recognition task which is very important for law enforcement agencies. The 
aim of this work, which allows real face photographs to be obtained using Sketch, is to identify real face photographs of possible offenders and thus capture them in a shorter time. For this purpose, sketch features are automatically obtained by RCNN. The RCNN features are then converted to their original dimensions using deconvolutional layers. During training, the images obtained at the network output are compared with the ground truth images and the network is updated. In the test phase, the images obtained at the network output are compared with the images stored in the data set. In this process, target image is matched with an image with the greatest SSIM. Images that remain under a certain SSIM value are not matched with any images in the database. The CUHK Face Sketch Database (CUFS) is used for training and testing of the proposed convolutional neural network architecture [14].

The rest of this work is organized as follows; In Section 2, problem definition, proposed CNN structure and data augmentation technique are examined. In addition, the details of the proposed method are explained. The dataset, experiments and experimental findings are presented in Section 3. The results are discussed in Chapter 4.

\section{Methodology}

\subsection{Problem Definition and Overwiev of Proposed Method}

Face detection is quite complicated process due to many challenging reasons such as facial look variations, expression and illumination changes. However, sketch matching is the addition of new challenges to all these challenges. In the face sketch process, the sketch is made according to the definition of a witness. In this case, all facial expressions depend on personal interpretation. The sketch-face recognition process, which is crucial for criminal investigation, can even vary depending on the psychological state of the examiner. In this case, face recognition becomes extremely difficult. Another difficulty is the ability to distinguish sketch drawings that are automatically generated by computers. To distinguish fake images will always be challenging. All these difficulties are quite misleading and confusing for the human eye. It is possible to deceive artificial intelligence methods using mug shots. For this reason, it may be a solution to obtain possible face photographs from sketch images and compare them to the database. In the comparison process, a similarity confidence coefficient must be specified. This confidence coefficient can be used to avoid possible counterfeit drawings.

Sketch images are applied to the proposed network entrance. At the exit of the network, RB face images are obtained. The main problem here is that the obtained facial photograph can be matched with the images on the dataset. The problem mentioned is shown in Figure 1.

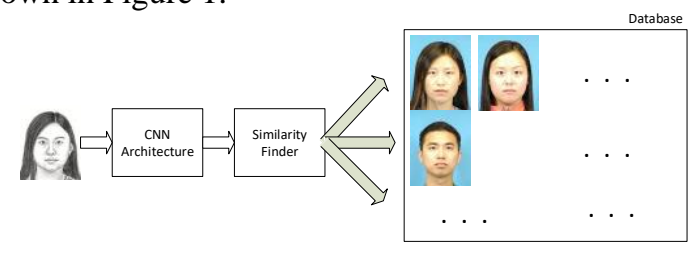

Figure 1. Sketch-Face recognition problem

The CNN architecture in Figure 2 has been proposed to solve this problem. The sketch image applied to CNN input is obtained as face photograph after 6 convolution, 6 ReLU, 4 pooling and 2 deconvolution layers.

\subsection{Data Augmentation}

In order for a network to be able to be trained strongly, the number of training cases must be high. The lower limit and upper limit of the number of training samples can not be predicted precisely, but the more the training sample, the lower the standard deviation. Especially the number of parameters in $\mathrm{CNN}$ constructions is rather high.

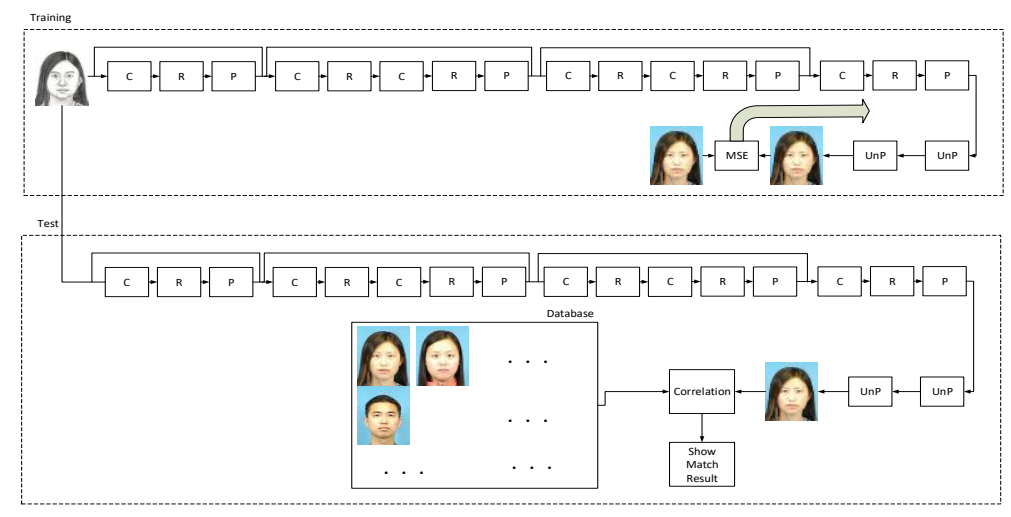

Figure 2. Proposed Method (C: convolution layer, R: ReLU, P: pooling layer, UnP: unpooling layer, MSE: mean square error) 
As the number of parameters increases, the number of training instances required must increase. Otherwise all parameters cannot reach optimum value. Nowadays, the spread of digital platforms creates a lot of data. However, these data must be labeled to be used in the training process. Tagging of images is very costly. Labeling of some images requires expert knowledge. For many medical and forensic applications, finding data is problematic. For all these reasons, the labeled data at hand should be used in the best possible way. For this, translation, deformation, reflection process etc. are applied to the training images.

In this study, the total number of images in the whole database is 188 . This data needs to be divided into 3 different classes as training, validation and testing. After this discrimination, 148 images remain for training. With so few images it is impossible to generalize the sketch-face recognition problem.

Also, it is aimed to provide data diversity in the training process by producing virtual data with the data augmentation method. With the developing technology, it will not be possible to encounter augmented images that we have increased data in real life. Due to the low number of images in the data set used alone, our network may not show high performance. We need to increase our diversity in order to get rid of this problem. For this reason, training data is multiplied by 3 with two augmentation techniques. As the first augmentation technique, a gaussian noise was added to the images. For this operation, the 2D gaussian operation is applied to the image as in Equation 1. $\sigma$ parameter in Equation 1 is selected as 2 .

$$
G(x, y)=\frac{1}{2 \pi \sigma^{2}} e^{-\frac{x^{2}+y^{2}}{2 \sigma^{2}}}
$$

As the second data augmentation technique, the salt \& pepper noise was added to all the images. For this purpose, noise is added randomly to the noise with a certain value. This noise is added to some images in color and some images in black and white. As the salt \& pepper noise value, twenty-one of the total number of pixels of the view is applied. This is to create distortion in some sections without disappearing the outline of the aimed image. Thus, in the training process, generalization is achieved.

\subsection{Proposed CNN Structure}

Convolutional neural network architecture is very inspiring for the solution of image processing problems. Deep nets can often learn low, middle and high level features related to the problem. But deep networks have some problems. As the network depth increases, the learning process becomes saturated. This saturation confirms the network memorization problem. In this case, as the training value continues to decrease, the validation accuracy value decreases. In some cases, the training accuracy does not decrease in the curve. More training can be used to overcome this problem. However, it is difficult to find more labeled training data. Residual structures are used to solve this problem in deep networks. In residual structures, the data at the entry of the layer is added to the last layer after processing. At this point, basic information about the data is not lost. Thanks to the residual structure, main image features from first layers are carried through to last layers. In this way, gradient vanishing problem is solved, and main features are protected. For example, eyes and brow structures are protected thanks to this architecture.

The proposed CNN architecture is basically a residual CNN structure. At the end of the network, the deconvolution structure is added. In this way, the original face images can be created. In the first part of the CNN structure, 6 convolution layers, 6 ReLU layers and 4 pooling layers are used. All convolution layers in the structure have $3 \times 3$ windows and the stride parameter is set to 1 . Convolution is applied as in Equation 2 [15].

$$
I_{i}^{l}=f\left(\sum_{j} I_{j}^{l-1} \otimes w_{i j}^{l}+b_{i}^{l}\right)
$$

Where I represents the image, w represents convolution windows, $\mathrm{b}$ represents bias parameter, $\mathrm{i}$ and $\mathrm{j}$ represent row and columns. The task of the ReLU layer is to prevent the network from being linear. For this reason, a very simple process is applied. Network parameters with negative values are assigned as 0 . The pooling layer is usually used to reduce the spatial dimensions of the image by down sampling. The max-pooling layer is used in this study. The Max-pooling layer selects the parameter with the greatest value from the pixels covered by the pooling window. At this point, the image dimensions are reduced to the window dimensions. Max-pooling is seen in Equation 3 [15].

$$
P_{j m}=\max _{k=1}^{r}\left(I_{j(m-1) n+k}\right)
$$

Where $\mathrm{P}$ represents pooling output, I represent input image of pooling layer, $\mathrm{j}$ and $\mathrm{k}$ represent dimension of area. When designing new CNN architectures, the image dimensions at the output of each layer should be appropriate for the next layer. For this reason, the dimensions of the image to be formed at the exit of each layer must be calculated. Stride, padding and window size affect the size of the image to be formed. Equation 4 is used to calculate the size of the image at the output of the convolution layer. Window size is size of kernels which are used 
in each layer. Equation 5 is used to calculate the viewing dimensions at the exit of the pooling layer.

$$
\begin{gathered}
P_{c}=\frac{S(\operatorname{Dim}-1)+w-I}{2} \\
P_{p}=\frac{\left(w_{p}-1\right)}{2}
\end{gathered}
$$

where, $\mathrm{P}_{\mathrm{c}}$ represents convolution layer output dimensions, $\mathrm{P}_{\mathrm{p}}$ represents pooling layer output dimensions, $S$ represents stride value, Dim represent input dimensions, w represents windows dimensions, I represent input image, $\mathrm{w}_{\mathrm{p}}$ represent pooling window size. Finally, the resulting feature matrices must be reinstated. For this reason, unpooling is applied to all matrices in the network output. The task of the unpooling layer is to do the opposite of pooling. In this process, image sizes are increased according to stride, padding and window size. The generated face image is compared with the ground truth image. The network is trained with the mean square error obtained for each pixel. For this purpose stochastic gradient descent method is used.

The proposed CNN architecture is basically a residual CNN structure. At the end of the network, the deconvolution structure is added. In this way, the original face images can be created. In the first part of the CNN structure, 6 convolution layers, 6 ReLU layers and 4 pooling layers are used. All convolution layers in the structure have $3 \times 3$ windows and the stride parameter is set to 1 . Convolution is applied as in Equation 2 [15].

In the test process, test images are applied to the network input in a sequence. The network produces a face photo for each sketch. The obtained image is subjected to standard single Structural Similarity Index (SSIM) process with all face photographs in the data set. SSIM measures perceptual differences between two images. SSIM is calculated using three terms; luminance, contrast and structure. The result is a multiplicative combination of these three terms. By selecting the image with the highest SSIM coefficient, the detection of the possible suspect is performed. If the SSIM coefficient is below the threshold value (similarity confidence coefficient), a warning is generated that there is no such face image in the dataset.

\section{Experimental Results}

\subsection{Data}

Proposed method is applied on a computer with Intel Core i7-7700K CPU (4.2 GHz) and NVIDIA GeForce GTX 1080 graphic card. The CUHK Face Sketch Database (CUFS) is used to train and test the proposed system. This set includes 188 sketch and face images. Of these images, 148 were randomly selected for training. Then 148 image data augmentation operations are applied. On this count, the training data is multiplied. Image resolutions are 200x250 pixels. All images are 3 channels (Red, Green, and Blue). Some images in the data set used are shown in Figure 4. Figure $4 \mathrm{~b}$ shows the original images in the dataset and the images obtained by data augmentation.

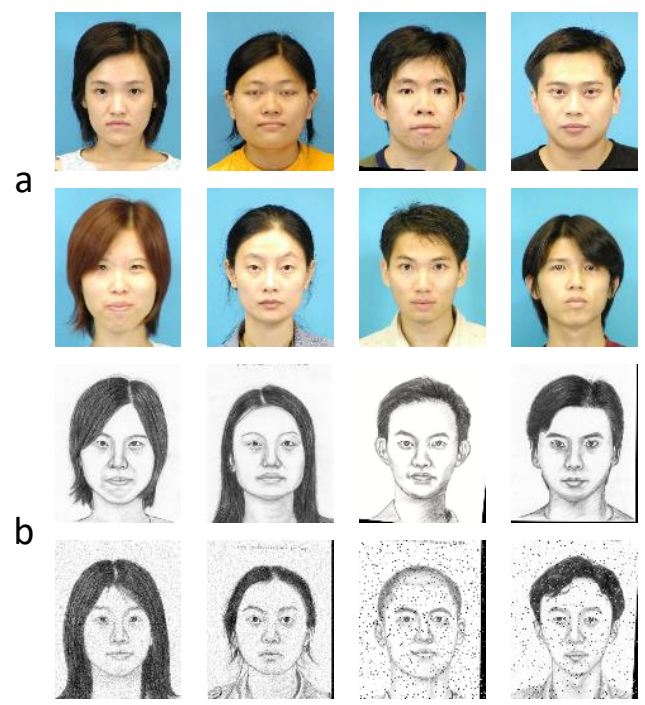

Figure 3. Image Dataset. a) sketch images, b) face images

\subsection{Experimental Results}

Sketch images for network training are divided into mini-batches. Training the network with a minibatch reduces the fluctuation in the learning curve and increase the ability of generalization. For this purpose, sketch images in the dataset are applied to the network in mini-batches consisting of 10 images. The network parameters are updated with the total error for each mini-batch. All convolution layers in the proposed architecture consist of $3 \times 3$ windows. In addition, the stride values are set to 1 and the padding parameter is set to 0 . In the first convolution layer, the depth of the sketch image (R, G, B) consisting of 3 layers is increased to 512. Then, this depth is increased to 1024 and reduced to 3 in the last layer. All pooling layers in the proposed CNN structure have $2 \times 2$ windows. The stride value of all pooling windows is set to 1 and padding is not applied. Increasing the stride values of the pooling layers causes further reduction of the image dimensions. This process, which is very successful for classification operations, can damage the image very much for retrieval systems. In the unpooling section, the image is converted to its original dimensions. For this reason, the image is enlarged with two unpooling layers. Residual part is the same as classical residual structure. The information at the output of the previous layer is added to the output of the next layer. 
The proposed network training and validation curves are shown in Figure 5. The training was stopped in 1000 epochs to understand training success. When the curves are examined, a steady decline indicates that the learning process is successful. Increasing the number of Epoch is not likely to disturb the learning behavior of the network. From here it is possible to ascertain that the number of samples is sufficient. The steady continuation of the slope between the training and validation curves indicates that there is no memorization. A dropout of 0.3 was used in the training. Thus, each parameter of the network can be determined effectively. This is why the training curriculum is a little lower in success.

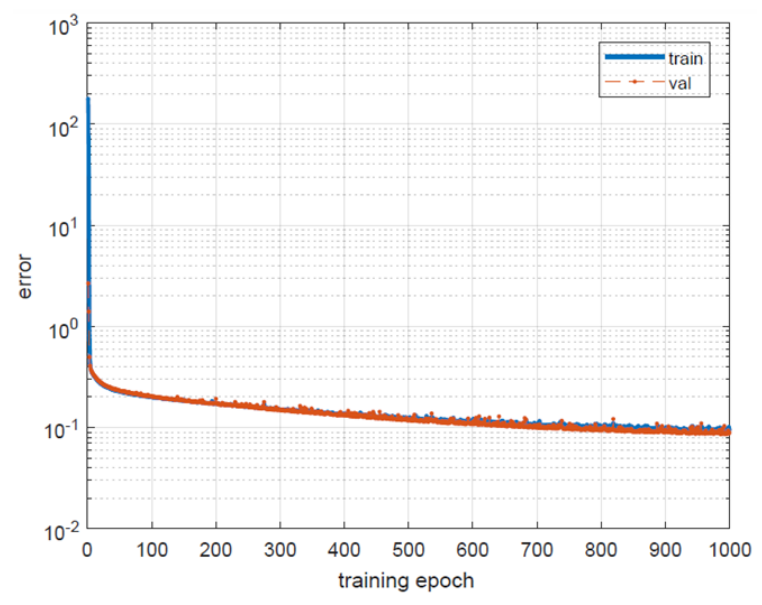

Figure 4. Training and validation curves of proposed $\mathrm{CNN}$ structure

According to Figure 5, the success of the training curve is $90.55 \%$ and the validation success is $91.1 \%$. When these images are examined, human faces can be perceived with outline. For color images we have just used, the actual images in the data set have been used. These are the virtual images that we have produced with the actual data increase. Due to the developments in technology, we are unlikely to meet augmented images in real life. For this reason, only color images obtained from actual data set images are visualized in the study.

In particular, basic features such as mouth, nose and eyebrows are quite evident. However, the eye structure is not very distinctive. To overcome this problem, an increase in the number of training samples and a deeper network structure can be offered as a solution.

Finally, the face images generated by the proposed network should be specified in the data set. For this purpose, the relation between all the images in the dataset produced is examined. The SSIM coefficient can produce similarity information. For this purpose, SSIM processing is applied to each image in the data set. The image pair that produces the highest SSIM coefficient is matched. But here a fundamental problem arises. The image that produces the highest SSIM coefficient in the entire data set is matched with the sketch. But this is a bad match. A threshold value has been set in order to avoid such fundamental problems. In order to determine the SSIM threshold, the correlation between all face images produced by the CNN structure and the ground truth face images is examined. The smallest of these SSIM values is chosen as the SSIM threshold value. If the face image produced by a sketch image is correlated with all the images in the data set and the generated SSIM value is lower than the threshold value, then we can come to the following conclusion: that person is not registered in our dataset.

The SSIM uses the distance function as the basis for similarity between statistical features between images. Unlike Corr2, the SIMM similarity function does not need to be gray level, and Corr2 uses histogram similarity as the similarity criterion. They obtain a theoretical criterion by obtaining the statistical and theoretical properties of the histogram. The Corr2 similarity criterion applies only to gray or binary images. It also cannot be used in RGB images. In this study, RGB images were obtained with RCNN structure from face sketch images. The use of SIMM and Corr2 in Binary images further reduces accuracy. Brcause, feature loss occurs during the conversion of RGB images to binary images.

The SSIM curve used in the determination phase is shown in Figure 5. According to Figure 8, the rate is $93.89 \%$. SSIM is applied to the images for the face detection from the database, which is the second step of the proposed system.

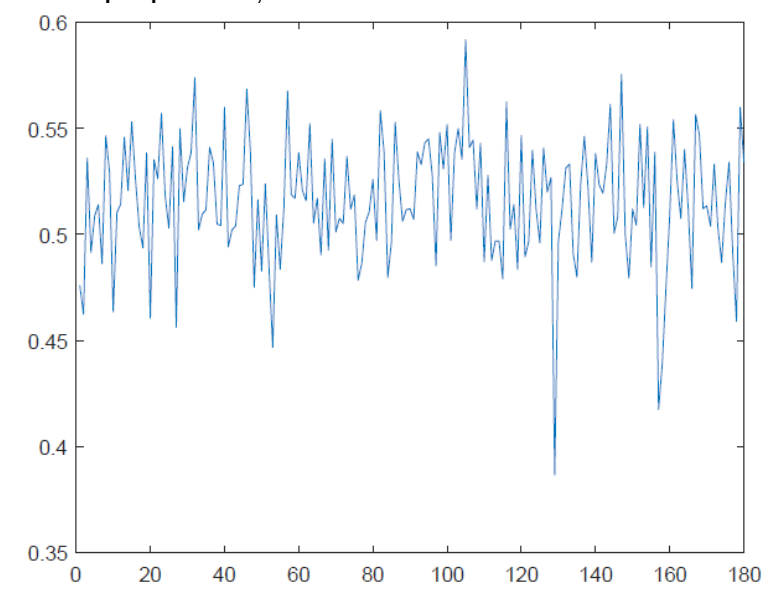

Figure 5. Face SSIM curve

Face images that are correctly determined and face images that are incorrectly specified were used to measure the success of the determination of the obtained face images in the data set. Accordingly, 176 images out of 188 images are correctly displayed. The remaining 12 face images were paired with the wrong persons.

As a result of the experiments performed, the correlation threshold value was set at 70 . The correlation curve used in the determination phase is shown in Figure 9. According to Figure 9, when the 
threshold value is set to 70 , the error rate is $5.5 \%$. Correlation is applied to the images for the face detection from the database, which is the second step of the proposed system.

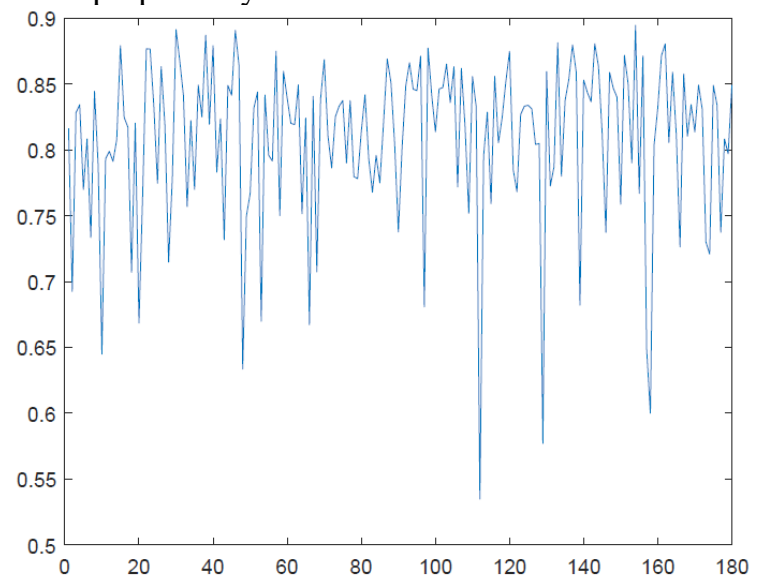

Figure 6. Face corelation curve

Correctly determined face images and incorrectly specified face images were used to measure the success of the determination of the obtained face images in the data set. Accordingly, 176 images out of 188 images are correctly displayed. The remaining 12 face images were paired with the wrong persons.

If we use Corr2 similarity function, correctly determined face images and incorrectly specified face images were used to measure the success of the determination of the obtained face images in the data set. Accordingly, 143 images out of 188 images are correctly displayed. The remaining 45 face images were paired with the wrong persons. In this case, the matching performance of the proposed method is roughly determined as $76.06 \%$.

Table 1 shows a fair success comparison between Corr2 function and SSIM similarity function.

Table 1. Corr2 and SSIM Comparison

\begin{tabular}{|l|l|l|l|}
\hline Method & $\begin{array}{l}\text { True } \\
\text { Faces }\end{array}$ & $\begin{array}{l}\text { False } \\
\text { Faces }\end{array}$ & Accuracy Rate \\
\hline Corr2 & 143 & 45 & $76.06 \%$ \\
\hline SSIM & 176 & 12 & $93.89 \%$ \\
\hline
\end{tabular}

Generally, RGB images are used in the literature to create face sketch images. In our study, it consists of RGB images from face sketch images and use similarity. Wang et al. proposed bayesian frameworkbased algorithm for creating face sketch images. Thanks to the prominent method, it achieves $97.48 \%$ accuracy [16]. Galoogahi et al. used gabor features based on a floating-point identifier. They achieved a high success rate of $99.91 \%$ in the CUFS and CUFSF data sets [17]. Zang et al. applied sparse representation is based on greedy search algorithm in order to be able to identify face sketch images. They used SSIM and FSIM as similarities measurements. The accuracy rates obtained for the CUHK data set are $99.6 \%$ (for
SSIM) and 92.86\% (FSIM) [18]. Güçlütürk et al. propose convolutional sketch inversion method. Their CUHK dataset SSIM result is 0.5658 [19]. Their results are based on similarity of images. But our results based on retrieval from dataset. Putri and Fanany proposed a sketch inversion technique. Their method is very strong RGB image creation method. Their image similarity parameter is 0.66 for SSIM [20]. Their results are based on similarity of images, too. Face sketch images are obtained from colored images throughout the mentioned studies. Our proposed method is to obtain color images from face sketch images. Although the method is different, it may be pointless to compare the results. When past studies are examined, accuracy achieved with the faceted sketch images varied between $97.48 \%$ and $99.6 \%$. In our proposed method, our accuracy is $93.89 \%$ with the obtained color images. This difference in accuracy is due to the fact that the methods used are completely different from each other.

\section{Conclusion}

In this study, face images from a sketch image were detected in a dataset. For this purpose, a CNN structure with residual architecture is proposed. The deconvolution unit is added to the output layer of the proposed $\mathrm{CNN}$ structure so that the original image dimensions can be obtained. Finally, the correct person in the dataset is determined by the SSIM method. In the first step of the proposed method, color face images are obtained from sketch images. The training and validation success in this section is quite high. The proposed network structure has passed a successful training process even though the number of labeled samples is limited. In the second part, the SSIM coefficient is used in the image matching part of the dataset. In future studies, the proposed network structure will be improved.

\section{References}

[1] Cubero, S., Lee, W. S., Aleixos, N., Albert, F., \& Blasco, J. (2016). Automated systems based on machine vision for inspecting citrus fruits from the field to postharvest-a review. Food and Bioprocess Technology, 9(10), 1623-1639.

[2] Hjelmås, E., \& Low, B. K. (2001). Face detection: A survey. Computer vision and image understanding, 83(3), 236-274.

[3] Jin, K. H., McCann, M. T., Froustey, E., \& Unser, M. (2017). Deep convolutional neural network for inverse problems in imaging. IEEE Transactions on Image Processing, 26(9), 4509-4522.

[4] Krizhevsky, A., Sutskever, I., \& Hinton, G. E. (2012). Imagenet classification with deep convolutional neural networks. In Advances in neural information processing systems (pp. 1097-1105).

[5] Tang, X., \& Wang, X. (2004). Face sketch recognition. IEEE Transactions on Circuits and Systems for Video Technology, 14(1), 50-57.

[6] Liu, Q., Tang, X., Jin, H., Lu, H., \& Ma, S. (2005, June). A nonlinear approach for face sketch synthesis and recognition. In Computer Vision and Pattern Recognition, 2005. CVPR 
2005. IEEE Computer Society Conference on (Vol. 1, pp. 1005-1010). IEEE.

[7] Zhang, W., Wang, X., \& Tang, X. (2011, June). Coupled information-theoretic encoding for face photo-sketch recognition. In Computer Vision and Pattern Recognition (CVPR), 2011 IEEE Conference on (pp. 513-520). IEEE.

[8] Galoogahi, H. K., \& Sim, T. (2012, July). Inter-modality face sketch recognition. In Multimedia and Expo (ICME), 2012 IEEE International Conference on (pp. 224-229). IEEE.

[9] Mittal, P., Vatsa, M., \& Singh, R. (2015, May). Composite sketch recognition via deep network-a transfer learning approach. In Biometrics (ICB), 2015 International Conference on (pp. 251-256). IEEE.

[10] Zhu, Z., Luo, P., Wang, X., \& Tang, X. (2014). Recover canonical-view faces in the wild with deep neural networks. arXiv preprint arXiv:1404.3543.

[11] X. Tang and X. Wang, Face Sketch Recognition, IEEE Transactions On Circuits And Systems For Video Technology, Vol. 14, No. 1, January 2004.

[12] K, Bansode N, and Sinha P K. "Face Sketch Generation Using Evolutionary Computing." International Journal on Soft Computing, vol. 7, no. 4, 2016, pp. 01-10., doi:10.5121/ijsc.2016.7401.

[13] S. Pramanik and D.Bhattacharjee, An Approach: Modality Reduction and Face-Sketch Recognition,2013, ArXiv

[14] Wang, X., \& Tang, X. (2009). Face photo-sketch synthesis and recognition. IEEE Transactions on Pattern Analysis and Machine Intelligence, 31(11), 1955-1967.

[15] Öztürk, Ş., \& Akdemir, B. A convolutional neural network model for semantic segmentation of mitotic events in microscopy images. Neural Computing and Applications, doi: 10.1007/s00521-017-3333-9.

[16] N. Wang, X. Gao, L. Sun, and J. Li, Bayesian Face Sketch Synthesis, IEEE Transactions On Image Processing, Vol. 26, No. 3, March 2017.

[17] Galoogahi, H. K., \& Sim, T. (2012). Face photo retrieval by sketch example. Proceedings of the 20th ACM International Conference on Multimedia - MM 12. doi:10.1145/2393347.2396354.

[18] S. Zhan, X. Gao, N. Wang and J. Li, Robust Face Sketch Style Synthesis, IEEE Transactions On Image Processing, Vol. 25, No. 1, January 2016.

[19] Y. Güçlütürk., U. Güçlü, R. van Lier \& M.. A. van Gerven, Convolutional sketch inversion. In European Conference on Computer Vision (pp. 810-824). Springer, 2006

[20] V. K. Putri \& M. I. Fanany. Sketch plus colorization deep convolutional neural networks for photos generation from sketches. In 2017 4th International Conference on Electrical Engineering, Computer Science and Informatics (EECSI) (pp. 1-6), 2017. 